\title{
A HOLLOW FIBER MEMBRANE PROBE FOR THE MEASUREMENT OF THE HYDROGEN CONCENTRATION IN A LIQUID
}

\author{
R. D. HOLSTVOOGD, ${ }^{\dagger}$ J. A. VAN 'T HOF and W. P. M. VAN SWAAIJ ${ }^{\ddagger}$ \\ Department of Chemical Engineering. Twente University of Technology, P.O. Box 217, 7500 AE \\ Enschede, Netherlands
}

(First received 6 June 1989; accepted in revised form 10 August 1990)

\begin{abstract}
A new method is introduced to measure the hydrogen concentration in liquids by applying a polyethersulfone hollow fiber membrane as a probe. Hydrogen rapidly diffuses through the fiber wall after which it is carried to a thermal conductivity detector by an inert gas flow. Stationary measurements in a pure hydrogen atmosphere as well as in a silicone oil are conducted. Polyethersulfone was shown to be more suitable as fiber material for a selective hydrogen probe than polysulfone. From nonstationary measurements it could be concluded that the response time of the polyethersulfone probe is fast enough to monitor the hydrogen concentration during instationary processes which involve hydrogen, like gas-liquid or liquid-solid mass transfer and homogeneous/heterogeneous reactions.
\end{abstract}

\section{INTRODUCTION}

In industry and laboratories many hydrogen consuming processes, e.g. hydrogenation of hydrocarbons, oils, and fats, are carried out. There is a need for an accurate and fast-response dissolved hydrogen analyzer in order to get a better understanding of the different mass transfer limiting steps and reaction rates involved in these often heterogeneously catalyzed gas-liquid systems. Such an analyzer also might be useful for control purposes. In our laboratory we study absorption and desorption of hydrogen in metal hydride/silicone oil slurries, and require a rapid and accurate dissolved hydrogen detector. Andersson and Berglin (1982) have developed a hydrogen electrode analogous to the well-known membrane-covered oxygen electrode, here based upon anodic oxidation of hydrogen on $\mathrm{Pt}$ in concentrated sulfuric acid. Reliable results were obtained by them. However, our experiences with this electrode (Chemtech Electrodes, Goteborg, Sweden) applied to hydrogen detection in silicone oil were not satisfactory due to low reproducibility. Moreover, a problem with this probe used in organic solutions is that the aqueous ionic solution at the electrode is affected by diffusion of solute through the membrane resulting in decreased and varying electrode sensitivity. Therefore membranes and ionic solutions have to be replaced after some hours.

To avoid these problems we developed a dissolved hydrogen analyzer based on the high diffusion rate of hydrogen through hollow fiber membranes. In our set-up hydrogen diffuses through the wall to the inside of a polyethersulfone hollow fiber, where it is transported by a carrier gas to a thermal conductivity

†Present address: Shcll Nederland Chemie B.V., P.O. Box 7005,3000 HA Rotterdam, Netherlands.

IAuthor to whom correspondence should be addressed. detector. The present investigation is concerned with the study of the rate limiting step during the mass transfer of hydrogen from the liquid (silicone oil) through the membrane into the gas stream, and the response rate of the probes.

\section{THE FIBER MEMBRANE PROBE}

Polysulfone and polyethersulfone are thermoplasts and their glass transition temperature is $180-185^{\circ} \mathrm{C}$. They are formed into anisotropic hollow fibers by coagulation of a concentrated solution of polyethersulfone (e.g. P3500 of Union Carbide) in Nmethylpyrrolidone. The fibers used here have been formed by a wet-spinning process, and were made by the membrane research group of our chemical engineering department. The fibers have been tested by them up to differential pressures of $6 \mathrm{MPa}$. Through this spinning process the fibers have, for the greater part of the wall thickness, a very porous structure, as can be seen in Fig. 1.

The outside of this asymmetric fiber has a more dense structure, and forms the major separational layer. However, for gas separation purposes in general this dense top layer is still too porous. For these purposes the fibers are coated with silicone rubber in order to fill the pores in this layer. In the Monsanto PRISM-hydrogen separation process silicone-coated polysulfone hollow fibers are applied.

In our case, however, no silicone-coated fibers are applied, because the liquid phase, silicone oil, would penetrate into the silicone rubber causing extreme swelling of this material, and giving nonreproducible results. After many weeks of testing no oil was found to have penetrated through the membrane in spite of the existing very fine pores $(<1 \mathrm{~nm}$ ). Obviousiy the oil molecules are too large to diffuse through the membrane wall. The main difference between the polyethersulfone and the polysulfone fiber lies in the 


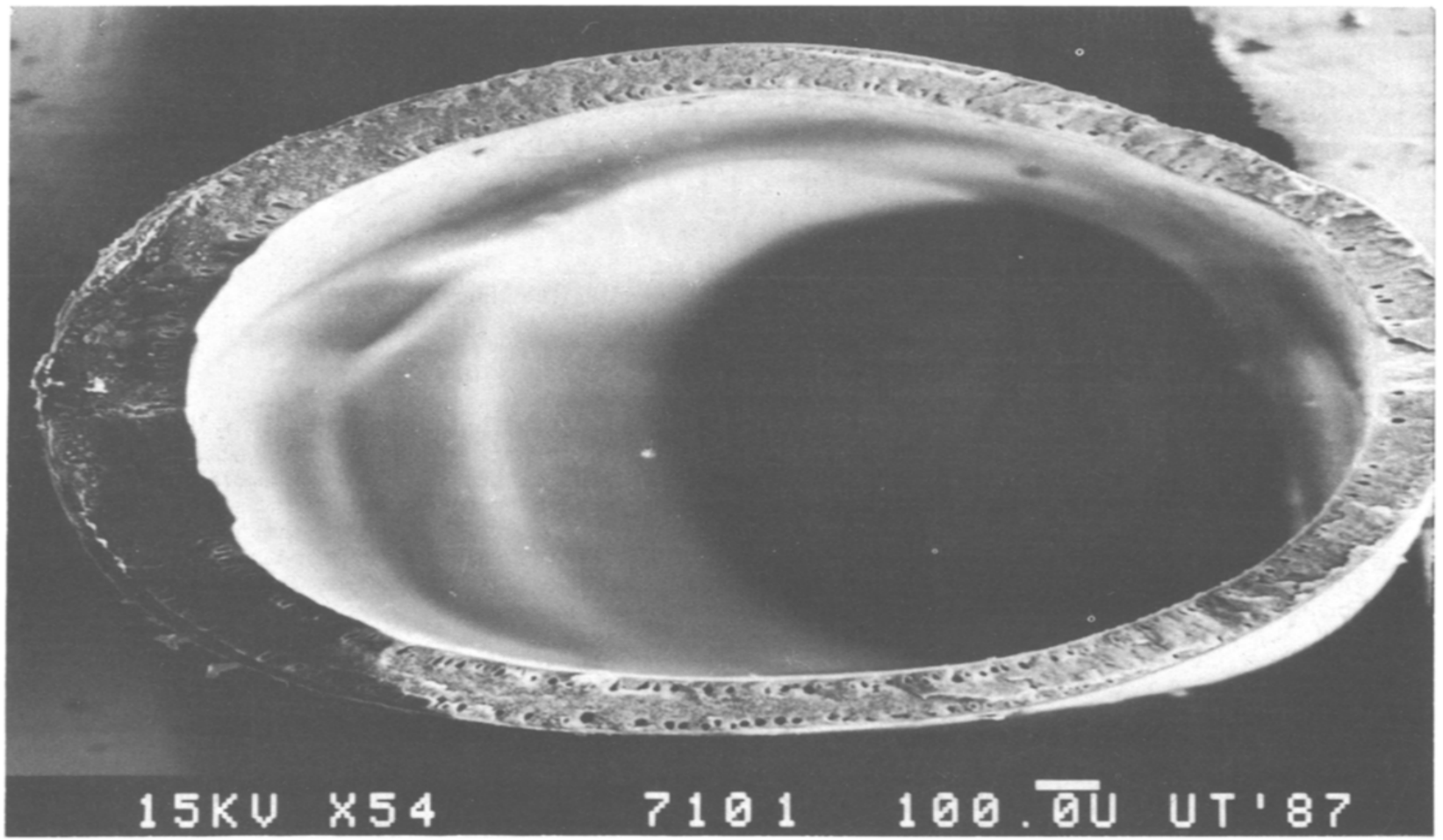

(a)

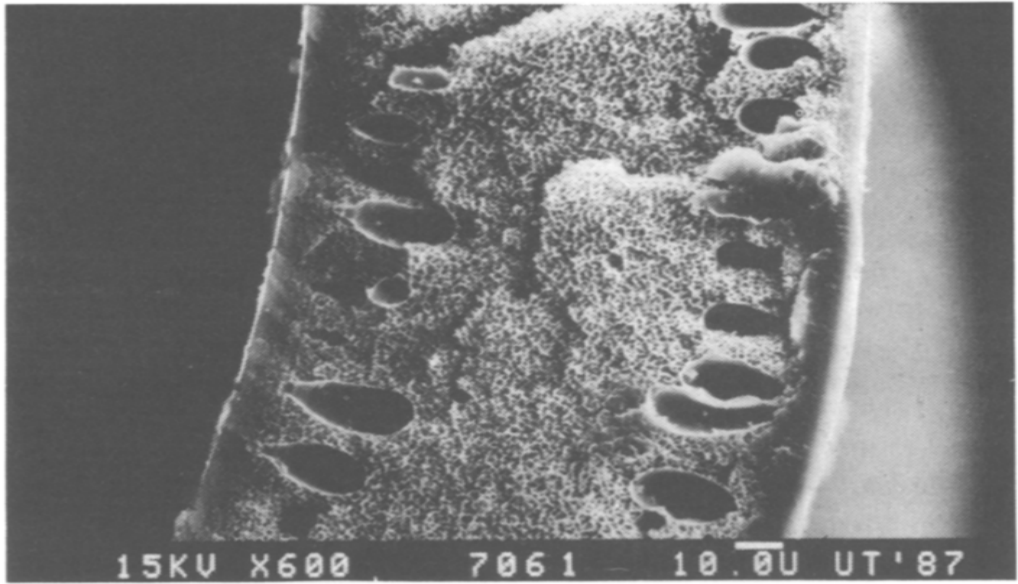

(b)

Fig. 1. Cross-sectional structure of a polysulfone hollow fiber.

structure of the membrane wall. The top layer at the outside of the polyethersulfone fiber membranc, which is very thin compared to the lower situated support layer, is much denser.

The newer polyethersulfone fiber has a much smaller diameter than the polysulfone fiber, which makes it more flexible and less breakable.

The fibers we used for our probe had the following dimensions:

$\begin{array}{lccc} & d_{f}, \mathrm{~mm} & d_{m}, \mathrm{~mm} & L_{f}, \mathrm{~m} \\ \text { Polysulfone } & 1.18 & 0.08 & 0.393 \\ \text { Polyethersulfone } & 0.60 & 0.08 & 0.170\end{array}$

The permeability of nonporous polysulfone for several gases has been investigated by Erb and Paul (1981). The permeability through a melt-extruded polysulfone film was found to decrease slightly with increasing gas pressure and is determined by the 
solubility and mobility/diffusion of the gas. At $35^{\circ} \mathrm{C}$ and $0.5 \mathrm{MPa}$ the solubility of $\mathrm{H}_{2}$ in polysulfone is: $m_{m}$ $=0.06$ (Erb and Paul, 1981; Chern et al., 1984). The hydrogen diffusion coefficient in nonporous polysulfone at $35^{\circ} \mathrm{C}$ and $2 \mathrm{MPa}$ is: $D_{m}=1.5$ $\times 10^{-11} \mathrm{~m}^{2} / \mathrm{s}$ (Chern et al., 1984).

The construction of the probe and experimental set-up is sketched in Fig. 2. One or more fibers are potted in a metal pipe, which is attached by a connector to the pipes of the in- and outflowing carrier gas streams. The oil used for the experiments was a silicone oil, Baysilone PD5 (Bayer), which has a low vapor pressure and a low viscosity as well (at $25^{\circ} \mathrm{C}$ $10^{-2}$ mbar and $3.7 \mathrm{cP}$ respectively). The hydrogen solubility in this oil at $25^{\circ} \mathrm{C}$ is: $m_{1}=0.081$, and is a function of temperature according to: $m=6.41$ $\times 10^{-2}+6.69 \times 10^{-4} T\left({ }^{\circ} \mathrm{C}\right)($ Holstvoogd, 1988)

\section{STATIONARY DIFFUSION CONDITIONS}

\subsection{Theory}

The stationary mass transfer of $\mathrm{H}_{2}$ through the porous membrane fiber into the carrier gas flow is shown schematically in Fig. 3. The first step is the dissolution of hydrogen in the top layer of the membrane (layer 1), followed by diffusion to the carrier gas side of the membrane. Usually the process of dissolution and diffusion of hydrogen is defined by the permeability of the membrane, $P_{m}\left(=m_{m} \times D_{m}\right)$. An overall permeability is defined because no distinction can be made between the permeabilities of the top and the support layer (layer 2). A distinction will be made between the permeability of gas-membrane and liquid-membrane systems respectively, $P_{m, g}$ and $P_{m, t}$, because parallel to dissolution in the top layer gas may also penetrate into the membrane through open pores, which can be partially filled by liquid.

The following overall mass transfer equation can be derived for transport of hydrogen from a pure hydrogen atmosphere through the membrane

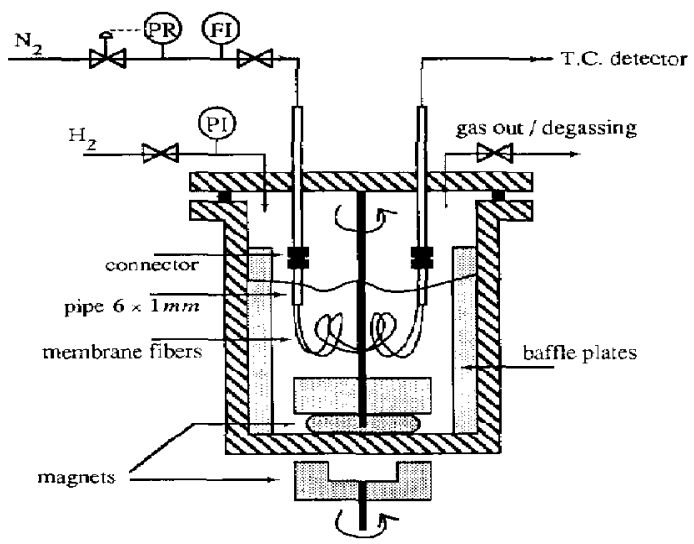

Fig. 2. Experimental set-up.
(Holstvoogd, 1988):

$$
\begin{gathered}
J=K^{G}\left(\bar{C}_{g, a}-\bar{C}_{g, i}\right) \\
\frac{1}{K^{G}}=\frac{d_{m}}{P_{m, g}}+\frac{1}{k_{g}} .
\end{gathered}
$$

The overall mass transfer coefficient is $K^{G}$. A similar equation can be defined in case the fiber is placed in a liquid, only then there will be an additional mass transfer resistance, the liquid phase to fiber mass transfer defined by $k_{t, f}$ :

$$
\begin{gathered}
J=K^{L}\left(\frac{\bar{C}_{I}}{m_{l}}-\bar{C}_{g, i}\right) \\
\frac{1}{K^{\mathbf{L}}}=\frac{1}{m_{l} k_{l, f}}+\frac{d_{m}}{P_{m, l}}+\frac{1}{k_{g}} .
\end{gathered}
$$

The overall mass transfer coefficients in the gas or oil phases, generally indicated by $K^{i}$, can be determined from the concentration of hydrogen outside the fiber, $C_{o}$ (when a gas phase is outside the membrane: $C_{a}=C_{g, a}, K^{i}=K^{G}$, or when there is an oil phase: $C_{o}$ $=C_{l} / m_{l}, K^{i}=K^{L}$ ), the measured hydrogen concentration in the carrier gas stream, $C_{g, i}$, and the total gas flow through the fiber. Assuming that through the fiber the carrier gas streams in plug flow, a differential mass balance can be made:

$$
\begin{gathered}
\frac{\mathrm{d}\left(\Phi_{g} C_{g, i}\right)}{\mathrm{d} z}-K^{i} \pi d_{f}\left(C_{\mathrm{o}}-\bar{C}_{g, i}\right)=0 \\
\text { at } \quad z=0: \bar{C}_{y, i}=0,
\end{gathered}
$$

the solution being

$$
\begin{array}{r}
\frac{1}{\left(1-X_{o}\right)^{2}} \ln \left[\frac{X_{o}\left(1-X_{i}\right)}{X_{o}-X_{i}}\right]+\frac{X_{i}}{\left(X_{o}-1\right)\left(1-X_{i}\right)} \\
=\frac{K^{i} \pi d_{f} L_{f}}{\Phi_{c}}
\end{array}
$$

and for

$$
X_{o}=1: \quad \frac{1}{2}\left[\frac{1}{\left(1-X_{i}\right)^{2}}-1\right]=\frac{K^{i} \pi d_{f} L_{f}}{\boldsymbol{\Phi}_{c}}
$$

\subsection{Results and discussion}

For possible mass transfer limitations inside or outside the fiber membrane, when the fibers were placed in a gas atmosphere or oil, it was checked if the

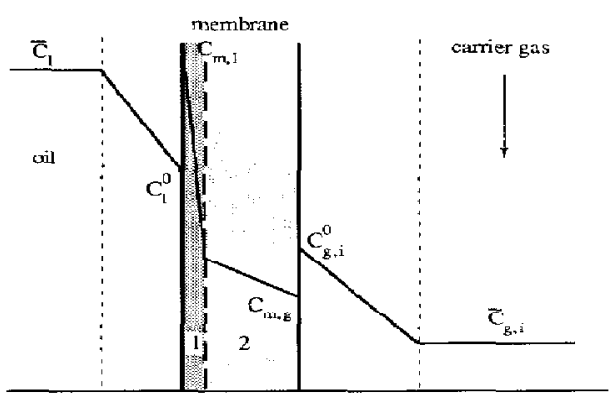

Fig. 3. Concentration profiles for mass transfer through membrane fiber wall, when the probe is submerged in liquid. 
carrier gas flow velocity and the stirrer speed had any influence on the overall mass transfer rate. From the experiments it could be concluded that for the polysulfone as well as for the polyethersulfone membrane fibers of carrier gas flow rate $0.5-2.0 \mathrm{ml} / \mathrm{s}$ and stirrer speeds above 300 r.p.m. had no influence on the absorption rate. Therefore the overall mass transfer rate is controlled by the membrane permeability. In eq. (6) plug flow of the carrier gas is assumed. This is correct, because the estimated Peclet number is larger than 2000 . The overall mass transfer coefficients can be calculated with eq. (6) from the experimental data: $C_{\theta, o}$ or $C_{t}, X_{i}$ and $\Phi_{c}$.

For both types of hydrogen probes the accuracy and reproducibility stayed good during the whole series of tests; no influence of the oil on the membrane material was experienced. The sensitivity of the probe is quite good, since it could detect at reduced hydrogen pressures above the oil hydrogen concentrations as low as $1 \mathrm{~mol} / \mathrm{m}^{3}$.

Polysulfone fiber membrane. Experiments with this membrane have been carried out in a pure hydrogen atmosphere up to pressures of $0.5 \mathrm{MPa}$. The measured permeability is independent of the hydrogen pressure outside the membrane. Its mean value at $25^{\circ} \mathrm{C}$ is:

$$
P_{m, g} / d_{m}=K^{G}=8.8 \times 10^{-6} \mathrm{~m} / \mathrm{s}( \pm 5 \%) .
$$

Comparison between the permeability of $\mathbf{H}_{2}$ through the polysulfone polymer known from the literature data and the permeability through the membrane shows that the permeability through the solid polymer is about a factor of 1000 smaller than the permeability through the membrane. This difference is due to the very porous structure of the membrane with the exception of the outermost layer, as can be seen in Fig. 1.

The experiments in the oil phase (PD5 silicone oil) have been conducted under different hydrogen gas pressures above the oil. Continuous dispersion of the $\mathrm{H}_{2}$ gas through the liquid was realized at different stirrer speeds and temperatures of $25-45^{\circ} \mathrm{C}$. The

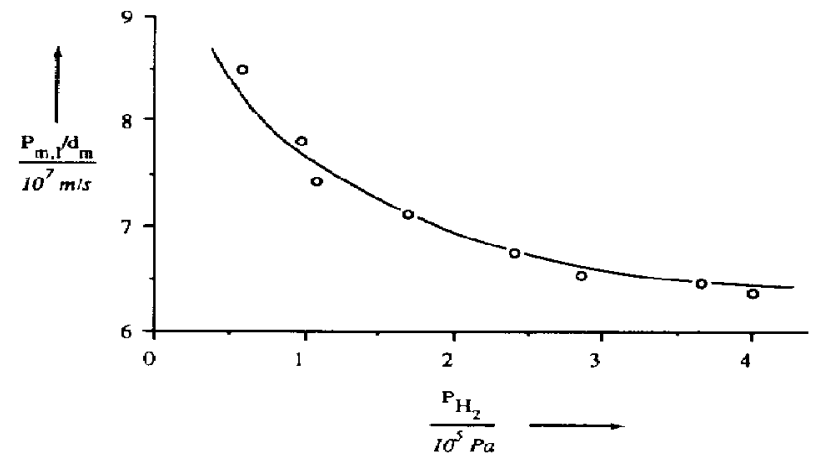

Fig. 4. The permeability of hydrogen through the polysulfone fiber in the presence of oil at different hydrogen pressures above the oil at $25^{\circ} \mathrm{C}$. calculated permeabilities at $25^{\circ} \mathrm{C}$ as a function of the hydrogen pressure above the oil are shown in Fig. 4. At low gas pressures the value of $P_{m, l}$ is ten times smaller than $P_{m, g}$, and slowly decreases with increasing pressure. The explanation for this behavior of the membrane in a liquid environment is that at increasing pressure at the surface of the membrane more pores are being filled with oil, and at the same time oil can penetrate further into the pores, thus increasing the diffusional resistance of the membrane.

Polyethersulfone membrane fiber. The overall gas mass transfer coefficient, $K^{G}$, has been measured as a function of the hydrogen pressure for temperatures up to $97^{\circ} \mathrm{C}$. Liquid phase measurements have been conducted at hydrogen (pure) pressures above the oil up to $1 \mathrm{MPa}$. In contrast to the polysulfone membrane fibers, here $P_{m, t}$ does not diminish with increasing pressure. Obviously the oil molecules cannot penetrate the dense top layer of this membrane as was the case for polysulfone.

The temperature effect on $P_{m, g} / d_{m}$ and $P_{m, l} / d_{m}$ is shown in Fig. 5 . As would be expected $P_{m, I}$ is equal to $P_{m, g}$, since no oil molecules could penetrate this membrane. The temperature dependence can be fitted by the following Arrhenius expression:

$$
P_{m} / d_{m}=7.589 \times 10^{-3} \exp \left\{\frac{-21.76 \mathrm{~kJ} / \mathrm{mol}}{R T}\right\} \mathrm{m} / \mathrm{s} .
$$

The gas permeability for the polysulfone fiber at $25^{\circ} \mathrm{C}$ and 1 bar is $7.1 \times 10^{-10} \mathrm{~m}^{2} / \mathrm{s}$. The permeability for the polyethersulfone fiber, $9.6 \times 10^{-11} \mathrm{~m}^{2} / \mathrm{s}$, at this temperature is smaller than for polysulfone, due to the dense top layer.

\section{NONSTATIONARY DIFFUSION CONDITIONS}

\subsection{Theory}

The response rate of the fiber probe (without detector) to a stepwise increase of the hydrogen concentration at the outside of the membrane can be determined by the time to establish a constant concentration profile over the membrane wall (diffusion penetration time) and/or by the time that is needed to reach a constant concentration gradient in the carrier

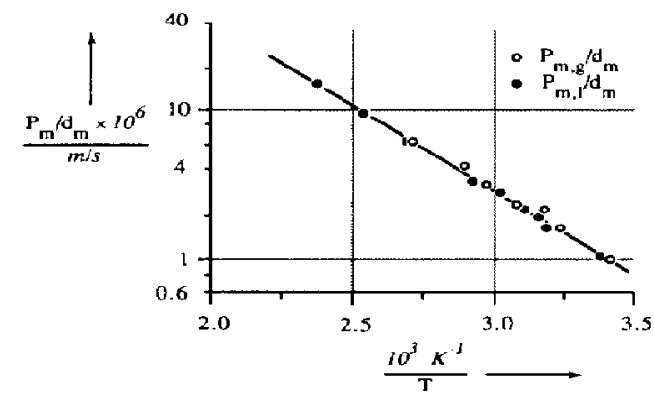

Fig. 5. Permeabilities of hydrogen through the polyethersulfone fiber vs reciprocal temperature. 

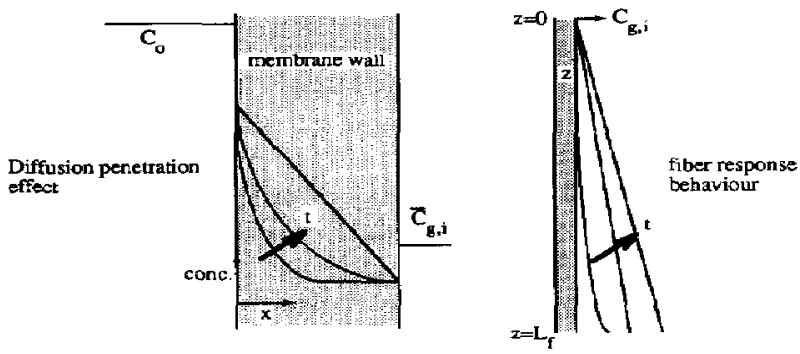

Fig. 6. Illustration of the nonstationary behavior of the fiber membrane probe.

gas stream along the fiber length. These two effects are elucidated in Fig. 6.

The fiber response effect is described by the following differential mass balance over a small section $\mathrm{d} z$ of the fiber, assuming quasi-stationary diffusion through the membrane, plug flow of the carrier gas in the fiber, and constant gas flow rate:

$$
\frac{\partial C_{i}}{\partial t}=-u_{g} \frac{\partial C_{i}}{\partial z}+\alpha\left(\frac{\bar{C}_{l}}{m_{l}}-C_{i}\right)
$$

and boundary conditions: $-t<0: C_{i}=0, \bar{C}_{i}=0$

$$
-t \geqslant 0: \bar{C}_{t}=\bar{C}_{l, t=0}
$$

with

$$
\alpha=\frac{P_{m}}{d_{m}} \cdot \frac{O}{A}=\frac{P_{m}}{d_{m}} \cdot \frac{4}{d_{f}} .
$$

The mean response time of a system is equal to the first normalized moment of the response on a step function (Westerterp et al., 1983) according to

$$
\bar{t}=M O_{1}=\frac{M_{1}}{M_{0}}
$$

The zero and first moment, $M_{0}$ and $M_{t}$ respectively, can be derived from the Laplace transformed differential mass balance, in the following way

$$
M_{n}=(-1)^{n} \lim _{s \rightarrow 0} \frac{\mathrm{d}^{n-1} \mathrm{~F}(z, s)}{\mathrm{d} s^{n-1}}=\int_{0}^{\infty} t^{n-1} F \mathrm{~d} t
$$

$$
\text { in which } \quad F=\frac{C_{i(a t z=L f)}}{\bar{C}_{l, t=0}} \text {. }
$$

Calculating $M_{1}$ and $M_{0}$ with the Laplace transformed solution of differential equation (7) and substituting these moments in eq. (9) results in the first normalized moment of the response curve for the fiber response effect only, $M O_{1, f}$ :

$$
M O_{1, f}=\frac{1}{\alpha}-\frac{\tau_{f} \mathrm{e}^{-\alpha \tau_{f}}}{1-\mathrm{e}^{-\alpha \tau_{f}}} .
$$

The nonstationary diffusion effect in the membrane wall will be discussed now. Assuming an overall permeability/diffusion coefficient in the membrane, the differential mass balance is described by

$$
\frac{\partial C}{\partial t}=-D_{m} \frac{\partial^{2} C}{\partial x^{2}}
$$

with boundary conditions:

$$
\begin{aligned}
& \text { - at } t=0, \forall x: C=0 \\
& \text { - at } x=d_{m}, \forall t: C=0
\end{aligned}
$$

for the condition at $x=0 ; t \geqslant 0$ two situations can be distinguished:

case 1. $C_{o}=m C_{g}$ or $C_{o}=C_{g}$

case 2. Limiting gas-liquid mass transfer, then (since

$$
\left.1 / K^{L} \gg 1 / k_{l}\right): C_{l}=m C_{g}\left[1-\exp \left[-k_{l} a t\right)\right] .
$$

The molar flux through the membrane in the case of very large $k_{l} a$ or pure hydrogen gas (case 1 ) is

$$
\begin{gathered}
\frac{J(t)}{J^{\infty}}=1+2 \sum_{n=1}^{\infty}(-1)^{n} \exp \left[-\left(\frac{n \pi}{d_{m}}\right)^{2} D t\right] \\
J^{\infty}=\frac{D C_{o}}{d_{m}} .
\end{gathered}
$$

with

Since the mean response time of the membrane, $M O_{\mathrm{L}, m}$ is defined by

$$
M O_{1, m}=\frac{1}{J^{\infty}} \int_{0}^{\infty}\left(J^{\infty}-J(t)\right) \mathrm{d} t
$$

the mean diffusion penetration time can be calculated from the molar flux solution, and becomes

$$
M O_{1, m}=\frac{d_{m}^{2}}{6 D_{m}}=\tau_{m} .
$$

In the case of gas-liquid mass transfer limitation by $k_{1} a$ (case 2), the reduced molar flux is

$$
\begin{aligned}
\frac{J(t)}{J^{\infty}}=1 & +2 \sum_{n=1}^{\infty}(-1)^{m} \exp \left[\frac{n^{2} \pi^{2} t}{6 \tau_{m}}\right] \\
& -\frac{d_{m} \sqrt{\frac{k_{1} a}{D}} \mathrm{e}^{-k_{l} a t}}{\sin \left[\sqrt{\frac{k_{l} a}{D}} d_{m}\right]} \\
& -2 \sum_{n=1}^{\infty}(-1)^{n} \frac{\exp \left[\frac{-n^{2} \pi^{2} t}{6 \tau_{m}}\right]}{\left[1-\frac{k_{l} a 6 \tau_{m}}{n^{2} \pi^{2}}\right]}
\end{aligned}
$$

in which the first two terms represent the flux for high $k_{1} a$.

However, the measured first moment at the detector will also include the response rate of the fiber, and the residence time of the gas in the pipe from the probe to the detector, $\tau_{p}$. The measured first moment is therefore

$$
M O_{1, d}=M O_{1, m}+M O_{1, f}+\tau_{p} .
$$

The experimental first moment after applying a step disturbance to the outside of the fiber can be calculated by integrating the difference between the concentration at stationary conditions, $C^{\infty}$, and the con- 
centration at time $t$. This relationship is derived from eqs (10) and (9), and results in

$$
M O_{1, d}=\frac{1}{C^{\infty}} \int_{0}^{\infty}\left(C^{\infty}-C(t)\right) \mathrm{d} t
$$

\subsection{Results and discussion}

Polysulfone membrane fiber. The step function in the hydrogen concentration in the oil was achieved by quick replacement of stripped oil by hydrogen saturated oil in the same experimental set-up as has been used for the stationary measurements.

The measured first moment at the detector has been plotted in Fig. 7 as a function of the reciprocal gas flow rate. The first moment of the fiber response, $M O_{1 . f}$ can be calculated with eq. (11), when the ratio $\alpha$ is known. The ratio $\alpha$ was determined with the permeability calculated from the stationary oil measurements, and is only dependent of the fiber properties: $1 / \alpha=d_{m} d_{f} /\left(4 P_{m, l}\right) \cong 344$ s. $M O_{1, f}$ is also plotted in Fig. 7 and represents the minimum response time of the total probe system, which is theoretically achievable for a certain carrier gas flow rate. It is small compared to the total detector response time. The residence time in the detector connection pipe $\left(\tau_{p}\right)$ could not easily be directly determined, but a volume of $5.4 \mathrm{ml}$ gave a good approximation of the calculated dependence of $M O_{1, d}$ with the reciprocal gas flow rate and the experimental slope of the curve in Fig. 7 and was not in conflict with the estimated actual connection pipe volume. Neglecting the response time of the fiber $\left(M O_{1, f}\right)$ we can extrapolate the measured line linearly to the vertical axis. The intercept of about $3 \mathrm{~s}$ is then a result of the instationary diffusion effect through the membrane. The diffusion penetration time $\tau_{m}$ can be estimated from the permeability, $\boldsymbol{P}_{\boldsymbol{m} \cdot l}$, and the hydrogen solubility in solid polysulfone known from the literature $\left(m_{m}=0.06\right)$. This gives a diffusion penetration time of about $1 \mathrm{~s}$, which is of the same order of magnitude as the experimental value. This is reasonable taking into account that by the presence of oil on and possibly in the membrane top layer the diffusion coefficient and the solubility in the membrane are influenced.

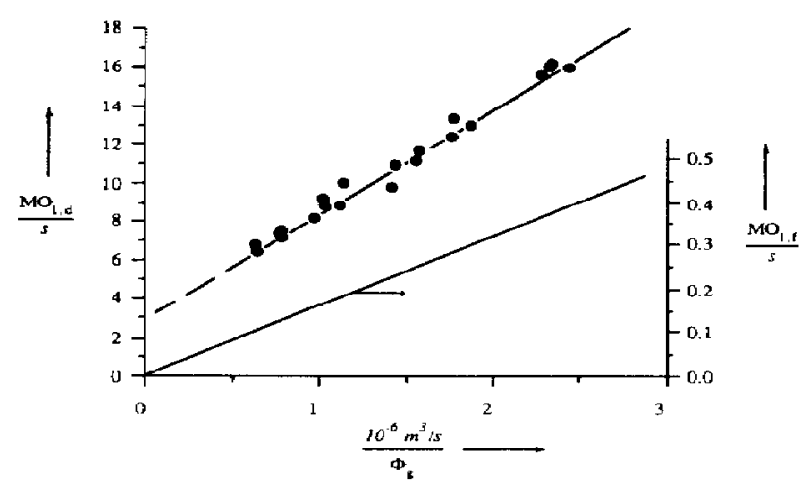

Fig. 7. $M O_{1, d}$ and $M O_{1, f}$ versus reciprocal carrier gas flow rate for the polysulfone fiber membrane at $25^{\circ} \mathrm{C}$.
Polyethersulfone membrane fiber. The mean diffusion penetration time has been determined from experiments in which the concentration response curve to a sudden increase of the gas pressure (no oil phase, case 1), since the presence of oil did not affect the permeability. For the applied fiber the response time of the fiber $\left(M O_{1, f}\right)$ was even smaller than the fiber response time of the polysulfone fiber, because this fiber has a much smaller diameter and is shorter, which makes the dimensionless factors $\alpha$ and $t_{f}$ smaller. Furthermore the residence time, $\tau_{p}$, to be eliminated from the total response could be measured, and amounted to $6.5 \mathrm{~s}$. After elimination of $\tau_{p}$ from the measured $M O_{1, d}$ the diffusion penetration times remain, and are plotted in Fig. 8 as a function of temperature $\left(20-100^{\circ} \mathrm{C}\right)$. The following relationship could be fitted:

$$
\tau_{m}=3.61 \times 10^{-2} \exp \left(\frac{14.27 \mathrm{~kJ} / \mathrm{mol}}{R T}\right) .
$$

The energy of activation of this process, which is mainly controlled by diffusion, is in the range of values that are usually found for diffusion processes.

Knowing the mean diffusion penetration time of the fiber one can measure the gas-liquid mass transfer rate in a reactor. With the probe we could successfully determine the volumetric mass transfer coefficient, $k_{l} a$. The employed method was that $k_{l} a$ in eq. (16) was varied until at every $t$ the theoretical ratio $J(t) / J^{\infty}$ fitted the measured reduced concentration at the detector, $C(t) / C^{\infty}$, which is equivalent to $J(t) / J^{\infty}$. Simultaneously to the probe measurements the $k_{l} a$ could also be determined by monitoring the falling hydrogen pressure after introduction of fresh hydrogen into the reactor, and calculating $k_{l} a$ from $k_{l} a=$ $-\ln \left[\left(P(t)-P_{t=\infty}\right) /\left(P_{t=0}-P_{t=\infty}\right)\right] / t$. For not too large $k_{l} a$ values good agreement was found between the $k_{1} a$ values of both methods at temperatures up to $150^{\circ} \mathrm{C}$. If $k_{t} a$ is high $\left(\gg 1 / \tau_{m}\right)$, the uncertainties in the determination of the response rate of the fiber introduce large errors in $k_{l} a$, because, then, the response of the probe is predominantly limiting the measured signal response rate instead of the nonstationary mass transfer process to be studied.

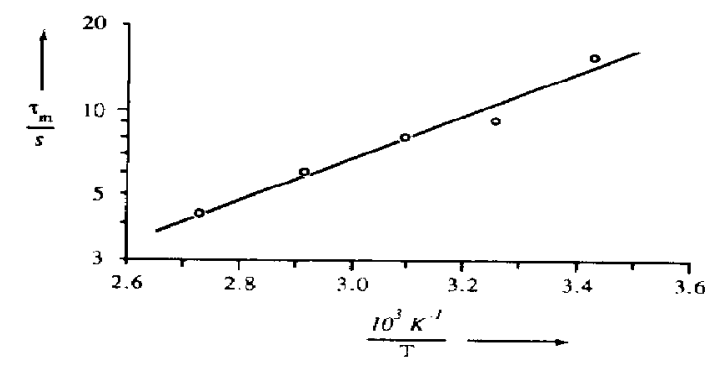

Fig. 8. Diffusion penetration time vs reciprocal temperature for the polyethersulfone fiber membrane. 


\section{CONCLUSIONS}

It has been demonstrated that the polyethersulfone hollow fiber membrane probe is a simple and reliable dissolved hydrogen detector. The developed hydrogen probe has proven to be accurate and gave over a longer period reproducible results combined with good sensitivity. The probe is only applied in an oil, but it can also be applied in different liquid systems unless the liquid affects the membrane properties. The polyethersulfone fiber membranes have been shown to be more suitable as fiber material for a selective hydrogen probe than polysulfone fiber membranes, because in the presence of a liquid phase no pressure influence of the overall mass transfer resistance could be detected in the studied pressure range (1-10 bar). The greater flexibility of the polyethersulfone fibers compared to the polysulfone fibers makes them less vulnerable.

The response rate of the fiber membrane probe was shown to be mainly limited by the diffusion penetration time through the membrane wall and the residence time between probe and detector. It has been demonstrated that instationary mass transfer and reaction processes in two- and three-phase dispersed systems can be followed with the fiber membrane probe developed here up to a temperature of $150^{\circ} \mathrm{C}$. If very short response times are required, the probe is less suitable.

Acknowledgements - These investigations were supported by the E.C., D.S.M. and the Foundation for Chemical Research in the Netherlands (S.O.N.). We also acknowledge the support of C. J. Visser, P. P. Jansen and G. Schorfhaar for their contributions to this work.

\section{NOTATION}

$A$ cross-sectional area of fiber, $\mathrm{m}^{2}$

$C$ hydrogen concentration, $\mathrm{mol} / \mathrm{m}^{3}$

$D_{m} \quad$ overall effective hydrogen diffusion coefficient through membrane, $\mathrm{m}^{2} / \mathrm{s}$

$d_{m} \quad$ membrane wall thickness, $m$

$d_{r} \quad$ diameter of fiber, $m$

$\boldsymbol{J}$ molar flux through the membrane, $\mathrm{mol} / \mathrm{m}^{2} \mathrm{~s}$ gas phase mass transfer coefficient in fiber, $\mathrm{m} / \mathrm{s}$

$k_{l, f} \quad$ liquid phase mass transfer coefficient outside membrane, $\mathrm{m} / \mathrm{s}$

$K^{G} \quad$ overall mass transfer coefficient, defined by eq. (2), m/s

$K^{L} \quad$ overall mass transfer coefficient, defined by eq. (4), m/s

$L_{f} \quad$ length of fiber, $m$

$m_{l} \quad$ solubility of hydrogen in oil $=C_{l} / C_{g}$

$m_{m} \quad$ solubility of $\mathrm{H}_{2}$ in the membrane

$M_{0} \quad$ zero moment, defined by eq. (10), s

$M_{1}$ first moment, defined by eq. (10), s

$M O_{1}$ normalized first moment, s

$O$ total pressure, $\mathbf{P a}$

$\boldsymbol{R}$

$s$

$t$

$t$

$T$

$u_{g}$

$X_{i}$

$\boldsymbol{X}_{0}$

$z \quad$ length coordinate, $\mathrm{m}$ permeability of hydrogen through membrane $=m_{m} D_{m}, \mathrm{~m}^{2} / \mathrm{s}$

gas constant $=8.1314 \mathrm{~J} /(\mathrm{mol} \cdot \mathrm{K})$

Laplace transformed time coordinate, $\mathrm{s}$

time, $s$

mean response time, $s$

temperature, $\mathbf{K}$

superficial gas velocity in fiber, $\mathrm{m} / \mathrm{s}$

molar fraction of hydrogen inside the fiber

gas core $=\bar{C}_{g, i}\left(\frac{R T}{P}\right)_{i}$

$X_{0}$ dimensionless $\mathrm{H}_{2}$ concentration outside

fiber $=C_{\circ}\left(\frac{R T}{P}\right)_{i}$

\section{Greek letters}

$\alpha \quad$ fiber constant, defined by eq. (8), 1/s

$\tau_{f} \quad$ residence time in fiber, $=\pi / 4 *\left(d_{f}\right.$

$\left.-d_{m}\right)^{2} * L_{f}, \mathrm{~s}$

$\tau_{m} \quad$ mean diffusion penetration time of membrane wall, $\mathrm{s}$

$\tau_{p} \quad$ residence time in line from probe to detector

$\quad=V / \Phi_{g}=L / u_{g}, \mathrm{~s}$

$\Phi_{g} \quad$ total gas flow $=\Phi_{c} /\left(1-X_{i}\right), \mathrm{m}^{3} / \mathrm{s}$

$\Phi_{c} \quad$ carrier gas flow, $\mathrm{m}^{3} / \mathrm{s}$

\section{Subscripts}

c carrier gas

$d \quad$ detector

$f \quad$ fiber

$y \quad$ gas phase or placed in gas

$i \quad$ at inside of fiber

$l \quad$ liquid phase or placed in liquid

$m$ membrane

o at outside of fiber

- Laplace transformed solution

\section{Superscripts}

0 at membrane surface

- in bulk

$\infty$

at stationary conditions

\section{REFERENCES}

Andersson, B. and Berglin, T., 1982, On the theory and use of a new fast-response dissolved hydrogen probe for hydrogen transfer studies. Chem. Engng J. 24, 201-212.

Chern, R. T., Koros, W. J., Hopfenberg, H. B. and Stannet, V. T., 1984, Mathematical selection for membrane-based gas separations. ACS Meeting, St. Louis, MO.

Erb, A. J. and Paul, D. R. 1981, Gas absorption and transport in polysulfone. J. Membr. Sci. 8, 11-22.

Holstvoogd, R. D., 1988, The absorption of hydrogen in metal hydride slurries, the influence of small solid particles on the gas-liquid mass transfer rate. Ph.D. Thesis, University of Twente, The Netherlands.

Westerterp, K. R., van Swaajj, W. P. M. and Beenackers, A. A. C. M., 1983, Chemical Reactor Design and Operation. John Wiley, New York. 